\title{
A Self-management Mechanism for Non-cooperative Behaviors in Large-scale Group Consensus Reaching Processes
}

\author{
Yucheng Dong, Sihai Zhao, Hengjie Zhang, Francisco Chiclana, and Enrique Herrera-Viedma
}

\begin{abstract}
In large-scale group decision making (GDM), non-cooperative behavior in the consensus reaching process (CRP) is not unusual. For example, some individuals might form a small alliance with the aim to refuse attempts to modify their preferences or even to move them against consensus to foster the alliance's own interests. In this paper, we propose a novel framework based on a self-management mechanism for non-cooperative behaviors in large-scale consensus reaching processes (LCRPs). In the proposed consensus reaching framework, experts are classified into different subgroups using a clustering method, and experts provide their evaluation information, i.e., the multi-criteria mutual evaluation matrices (MCMEMs), regarding the subgroups based on subgroups' performance (e.g., professional skills, cooperation, and fairness). The subgroups' weights are dynamically generated from the MCMEMs, which are in turn employed to update the individual experts' weights. This self-management mechanism in the LCRP allows penalizing the weights of the experts with non-cooperative behaviors. Detailed simulation experiments and comparison analysis are presented to verify the validity of the proposed framework for managing non-cooperative behaviors in the LCRP.
\end{abstract}

Keywords: large-scale group decision making, consensus reaching process, self-management mechanism, non-cooperative behaviors.

\section{INTRODUCTION}

Group decision making (GDM) $[18,27]$ can be viewed as a process in which a group of experts express their opinions regarding a decision problem and they aim at achieving a collective solution. Most existing GDM methods focus on how to obtain a collective opinion to a decision problem without addressing whether the consensus level among experts can or cannot be guaranteed. Thus, the consensus reaching process (CRP) is proposed and widely used to help experts improve the group consensus level $[19,23,70]$. Traditionally, CRP focuses on how to obtain a collective opinion with a full consensus. However, to obtain a full consensus is very time-consuming and difficult, and in many practical GDM problems it is unnecessary. Therefore, the concept of "soft" consensus level $[5,6,24,28,29,40]$ has been proposed and used widely in a variety of proposed CRPs: (1) with different preference representation formats $[7,9,11,26,58]$; (2) based on minimum adjustments or cost $[3,4,12,21,22$, 66]; (3) driven by consistency and consensus measures [17, 56, 57, 59]; (4) considering the attitudes of experts [39, 45, 61]; and (5) within dynamic/Web context [13, 15, 31, 43, 69].

With the development of information technology and society, decision contexts become more and more complex as large number of stakeholders are often involved. To provide support in large-scale GDM contexts, a number of decision models have been reported: Zhu et al. [71] proposed a hierarchical clustering approach for large-scale GDM; Wu and Liu [53] proposed an interval type-2 fuzzy clustering solution for large-scale multiple criteria GDM problems; Zhang et al. [68] proposed to manage multi-granular linguistic distribution assessments in large-scale multi-attribute GDM; Palomares [37] proposed an attitude-based consensus model for large-scale GDM in IT services management; Quesada et al. [44] proposed a large-scale consensus reaching framework with uninorm aggregation operators; Zhang et al. [67] proposed to build a consensus for the heterogeneous large-scale GDM; Wu et al. [54] proposed an interval type-2 fuzzy TOPSIS model for large-scale GDM problems with social network information; while $\mathrm{Wu}$ et al. [55] reported a linguistic solution for double large-scale GDM in E-commerce.

In a large-scale consensus reaching process (LCRP), because experts usually come from different domains and have different interests, they may adopt non-cooperative behaviors to further their own interests. For example, some individuals might

This work was supported by the grant (No. 71571124) from NSF of China, the grants (Nos.TIN2013-40658-P and TIN2016-75850-R) from the FEDER funds, and the grant (No.TIC-5991) from the Andalusian Excellence Project.

Y. Dong, and S. Zhao are with the Business School, Sichuan University, Chengdu 610065, China (e-mails: ycdong@scu.edu.cn (Y. Dong), shzhao@stu.scu.edu.cn (S. Zhao)).

H. Zhang is with the Business School, Hohai University, Nanjing 211100, China (e-mails: hengjiezhang@126.com, hengjiezhang@hhu.edu.cn).

F. Chiclana is with the Centre for Computational Intelligence, Faculty of Technology, De Montfort University, Leicester, UK (e-mail: chiclana@dmu.ac.uk)

E. Herrera-Viedma is with the Department of Computer Science and Artificial Intelligence, University of Granada, Granada, Spain, and as visiting professor with the Department of Electrical and Computer Engineering, Faculty of Engineering, King Abdulaziz University, Jeddah, Saudi Arabia (e-mail: viedma@decsai.ugr.es). 
form a small alliance and refuse to modify their preference or change their preference in a direction against consensus to further their alliance's own interests. Hence, managing experts' non-cooperative behaviors is key to assure the quality of the GDM outcome. In order to deal with non-cooperative behaviors in the GDM, two mainstream research approaches have been developed in the literature:

(1) Managing non-cooperative behaviors in the aggregation process (or selection process) of the GDM, which focuses on the influence of the non-cooperative behaviors on the aggregation outcome. For example, Pelta and Yager [42] investigated non-cooperative behaviors in the aggregation process in mediated multiagent negotiations via an optimization approach. Recently, Dong et al. [10] studied the non-cooperative behaviors, called strategic weight manipulation, in the aggregation process of the multiple attribute GDM. They showed that the performance of the ordered weighted averaging (OWA) operator [64] is better than the weighted averaging (WA) operator in defending against the strategic weight manipulation in the multiple attribute GDM. Additional studies regarding this topic have been reported by Yager in [62, 63].

(2) Managing non-cooperative behaviors in the consensus process of the GDM, which mainly analyzes whether a consensus solution can be achieved under the presence of non-cooperative behaviors in the CRP. For example, Palomares et al. [41] investigated the non-cooperative behaviors in the CRP of GDM and they designed a mechanism that depends on a moderator to manage non-cooperative behaviors. Moreover, simulation experiments reported by Palomares et al. [41] showed that their mechanism could improve the probability to achieve a consensus solution. Following this research methodology, Quesada et al. [44] suggested an approach to manage non-cooperative behaviors in the LCRP with uninorm aggregation operators, while Dong et al. [14] proposed a self-management mechanism for managing non-cooperative behaviors with was justified with extensive simulation experiments..

In the first mainstream research approach, theoretical analysis is often used to analyze non-cooperative behaviors in the aggregation process, while in the second one simulation experiments are used as a tool to analyze non-cooperative behaviors because the CRP is a complex and dynamic process, which makes the theoretical development challenging. By analyzing existing non-cooperative behavior studies in GDM models, we find the following research issues that should be addressed:

(1) Non-cooperative behaviors often appear in the CRP, which presents more complex characteristics than the aggregation process in GDM problems. However, models presented in $[10,42,62,63]$ only discuss the non-cooperative behaviors in the aggregation process in GDM problems.

(2) Models to manage non-cooperative behaviors in the CRP, presented in [41, 44], depend on a moderator. However, in practical decision situations, it is sometimes difficult for a moderator to effectively carry out such heavy and complicated task.

(3) The self-management mechanism in [14] manages non-cooperative behaviors based on mutual evaluation matrices in the CRP. However, in the LCRP the self-management mechanism cannot work because it is very difficult to manage mutual evaluation matrices among a large group of experts.

Motivated by the challenge to deal with non-cooperative behaviors in the LCRP, in this paper we continue the research line of [14] and propose a novel framework based on a self-management mechanism for managing non-cooperative behaviors in the LCRP. In the proposed framework, experts express their opinions, are classified into a number of subgroups using a clustering technique, and then they provide multi-criteria mutual evaluation matrices (MCMEMs) regarding the subgroups, which are used to derive subgroups' weights. Experts' weights will be updated dynamically using the obtained subgroups' weights, and integrated into the LCRP. In this way, the weights of the experts with non-cooperative behaviors will be penalized by the self-management mechanism in the LCRP.

The rest of this paper is organized as follows: Section II introduces the preliminaries. Section III proposes the LCRP framework based on a self-management mechanism. Section IV introduces several common non-cooperative behaviors. Also, within this section, detailed simulation experiments and comparison analysis, as well as an illustrative example, are presented to verify the validity of the proposed framework. Section V discusses the advantages and limitations of our proposal. Finally, conclusion and future work are covered in Section VI.

\section{PRELIMINARIES}

This section introduces some basic knowledge regarding the consensus reaching process with preference relations and the LCRP.

\section{A. The consensus reaching process with preference relations}

There are two basic elements in a GDM: a finite set of alternatives, $X=\left\{x_{1}, x_{2}, \ldots, x_{n}\right\}(n \geq 2)$; and a finite set of experts, $E=\left\{e_{1}, e_{2}, \ldots, e_{m}\right\} \quad(m \geq 2)$. Each expert can express his/her preference on $X$ using different preference representation structures, such as utility function [51], preference orderings [47], multiplicative preference relations [46, 48], additive preference relations (or fuzzy preference relations) [25, 36, 51], and linguistic preference relations [50]. Additive preference relations have been widely used in GDM. Without loss of generality, in this paper we assume that experts express their preferences using additive preference relations.

Definition 1 (Additive preference relation [36, 51]). An expert expresses his/her preferences on a set of alternatives $X$ by a matrix, $P=\left(p_{i j}\right)_{n \times n}$, in which $p_{i j} \in[0,1]$ denotes the preference degree of alternative $x_{i}$ over $x_{j}$. It is assumed that the preference relation satisfies the additive reciprocity property, $p_{i j}+p_{j i}=1, \forall i, j$. 
For simplicity, we call additive preference relations simply preference relations in this paper.

Many CRP models based on preference relations have been proposed in the specialized literature, which normally comprise a consensus process and a selection process.

(1) Consensus process that aims at improving the consensus level among experts [8,52], and it consists of two parts: consensus measure and feedback adjustment.

(a) Consensus measure is used to compute the level of consensus or agreement among experts. Different methods to measure consensus are possible [8, 38, 40]. In general, the computation of the consensus level in GDM is done in two ways: (1) measuring the similarity of preferences between pairs of experts; (2) measuring the similarity of preferences between individuals and the group as a whole. Following (1), a novel consensus measure method was developed by Palomares et al. [41] that implemented weights of experts. Without loss of generality, in this paper, we adopt this approach as described below.

For a pair of experts $\left(e_{k}, e_{l}\right)(k=1, \ldots, m-1, l=k+1, \ldots, m)$, a preference similarity matrix, $S M=\left(s m_{i j}^{k l}\right)_{n \times n}$, is defined, with element $s m_{i j}^{k l}=1-\left|p_{i j}^{k}-p_{i j}^{l}\right|$. Let $\omega_{k}$ and $\omega_{l}$ be the weights associated to experts $\left(e_{k}, e_{l}\right)$, respectively. The following consensus matrix, $C M=\left(c m_{i j}\right)_{n \times n}$, with element

$$
c m_{i j}=\sum_{k=1}^{m-1} \sum_{l=k+1}^{m} \omega_{k l} s m_{i j}^{k l} / \sum_{k=1}^{m-1} \sum_{l=k+1}^{m} \omega_{k l}
$$

and $\omega_{k l}=\min \left\{\omega_{k}, \omega_{l}\right\}$ is computed [41]. The consensus can be measured at the following three different levels:

(i) Consensus level on a pair of alternatives $\left(x_{i}, x_{j}\right)$,

$$
c p_{i j}=c m_{i j}
$$

(ii) Consensus level on alternative $x_{i}$,

(iii) Collective consensus level,

$$
c a_{i}=\sum_{j=1, j \neq i}^{n} c m_{i j} /(n-1)
$$

$$
c l=\sum_{i=1}^{n} c a_{i} / n .
$$

It is obvious that $c l \in[0,1]$. The higher $c l$ is the higher the consensus level among all experts is.

(b) Feedback adjustment is used to support experts modify their preferences to improve consensus level. Different feedback adjustment methods have been proposed [26,49]. In this paper, the adjustment method is used and described:

Let $P^{k}=\left(p_{i j}^{k}\right)_{n \times n}(k=1,2, \ldots, m)$ be the preference relation of expert $e_{k}, P^{c}=\left(p_{i j}^{c}\right)_{n \times n}$ be the collective preference relation, and $\overline{P^{k}}=\left(\overline{p_{i j}^{k}}\right)_{n \times n}$ be the adjusted preference relation of expert $e_{k}$. The adjustment process obeys following rules:

$$
\begin{cases}\overline{p_{i j}^{k}} \in\left[\min \left(p_{i j}^{k}, p_{i j}^{c}\right), \max \left(p_{i j}^{k}, p_{i j}^{c}\right)\right], & \text { if } i<j \\ \overline{p_{j i}^{k}}=1-\overline{p_{i j}^{k}}, & \text { if } i>j\end{cases}
$$

(2) Selection process aims to help experts find a solution to a decision problem. It is usually applied in two stages [26].

(a) Aggregation phase to derive the collective preference relation, $p^{c}=\left(p_{i j}^{c}\right)_{n \times n}$, where $p_{i j}^{c}$ denotes the group's preference degree of alternative $x_{i}$ over $x_{j}$. Usually, the WA operator is used in the aggregation phase, as it is the case in this paper:

$$
p_{i j}^{c}=\omega_{1} p_{i j}^{1}+\omega_{2} p_{i j}^{2}+\ldots+\omega_{k} p_{i j}^{k}+\ldots+\omega_{m} p_{i j}^{m}=\sum_{k=1}^{m} \omega_{k} p_{i j}^{k}
$$

where $\omega_{k} \in[0,1]$ is the weight associated to expert $e_{k}$ and $\sum_{k=1}^{m} \omega_{k}=1$.

(b) Exploitation phase to rank the alternatives. This is done by associating a collective preference value, $U_{i}$, to each alternative $x_{i}$. Several methods have been suggested to generate $U_{i}$, such as OWA operator and row averaging methods. In this study we use row averaging method to calculate $U_{i}$, i.e.

$$
U_{i}=\sum_{j=1}^{n} p_{i j}^{c} / n, i=1,2 \ldots, n
$$

The higher the value of $U_{i}$ is the better the alternative $x_{i}$ will be for the group of experts. According to Eq. (7), a ranking of alternatives can be obtained.

\section{B. Large-scale consensus reaching process}

Compared with traditional GDM with small group of experts, the LCRP problem involves large number of experts. Several methods have been proposed to cope with this type of problem in the existing literature, which usually obey the following scheme (e.g., [61, 67]): first of all, a clustering method is used to classify the large number of experts into different subgroups; then, a consensus process is utilized to improve the consensus level among experts; and finally, a selection method is used to 
obtain a collective preference.

From this analysis, it can be seen that the most prominent feature of LCRP is the use of a clustering process, with many clustering methods available to perform this task: K-means algorithm [35], Fuzzy clustering algorithm [2], Grey clustering algorithm [34] and Fuzzy C-Means algorithm [32]. The proposed consensus reaching framework is a general framework for the large-scale GDM, and the choice of the clustering algorithm will not change the essence of the framework. In this study we adopt the Grey clustering algorithm, which is summarized below altough readers are referred to Liu et al. [34] for its detailed description.

The Grey clustering algorithm is based on the similarity measure between experts, and the following two principles: given two experts $\left(e_{k}, e_{l}\right)$, if their similarity degree $s^{k l}$ defined as

$$
s^{k l}=\sum_{i=1}^{n} \sum_{j=1}^{n} s_{i j}^{k l} /\left(n^{2}-n\right)
$$

is greater than a given threshold $r(r \in[0,1])$, then they are considered neighbors; given a subgroup and an expert, if the proportion of experts in the subgroup who are neighbors of the expert is greater than a given threshold $c(c \in[0,1])$, then the expert can be added to the subgroup. The value of $r$ (or $c$ ) will have an effect on the number of clusters, with a higher value leading to a higher number of clusters. Thus, setting different parameters $r$ and $c$ values will not have an influence on the proposed large-scale GDM framework other than the different numbers of clusters to deal with. In the simulation experiments reported in this study, we set $r=0.75$ and $c=0.7$.

\section{The Large-Scale Consensus Reaching Framework Based On A Self-Management Mechanism}

This section proposes the LCRP problem, and formally presents its resolution framework based on a self-management mechanism.

\section{A. Large-scale consensus reaching process problem with non-cooperative behaviors}

As mentioned in Section I, the consensus building among a large number of experts has become a trend and hot topic of research. Non-cooperative behaviors exist in the LCRP, and therefore it is important to study it and to tackle it with appropriate theoretically based models. This is the aim of this study, where a large-scale consensus reaching problem with non-cooperative behaviors, which is described as below, is proposed:

Recall that $X=\left\{x_{1}, x_{2}, \ldots, x_{n}\right\}$ is a finite set of alternatives; $E=\left\{e_{1}, e_{2}, \ldots, e_{m}\right\}$ is a finite set of experts; $P_{i j}^{k}=\left(p_{i j}^{k}\right)_{n \times n}$ is the preference relation provided by expert $e_{k}$ on $X$; and $\omega=\left(\omega_{1}, \omega_{2}, \ldots, \omega_{m}\right)^{T}$ is a weight vector, where $\omega_{k}$ is the weight of the expert $e_{k}$ and $\sum_{i=1}^{m} \omega_{k}=1$.

In the decision process, experts may adopt non-cooperative behaviors to further their own goals. For example, some individuals might form an alliance that refuse to modify its preference or move its preference against consensus to further its own interests in the LCRP. Therefore, how to design a large-scale consensus reaching framework to cope with these non-cooperative behaviors is essential.

\section{B. The large-scale consensus reaching framework}

This section proposes a large-scale consensus reaching framework based on a self-management mechanism. The resolution of LCRP problems usually follows a scheme consisting of three different parts: clustering process, consensus process and selection process [41]. In the clustering process, an algorithm is applied to classify experts into different subgroups. The consensus process aims to improve the consensus level among experts, while the selection process focuses on obtaining a ranking of the alternatives.

By integrating experts' weights generated dynamically into the LCRP, we propose a novel framework based on a self-management mechanism. In the proposed framework, four parts are differentiated: clustering process, dynamically generating weights process, consensus process and selection process. The details of the framework are presented in Fig. 1. 


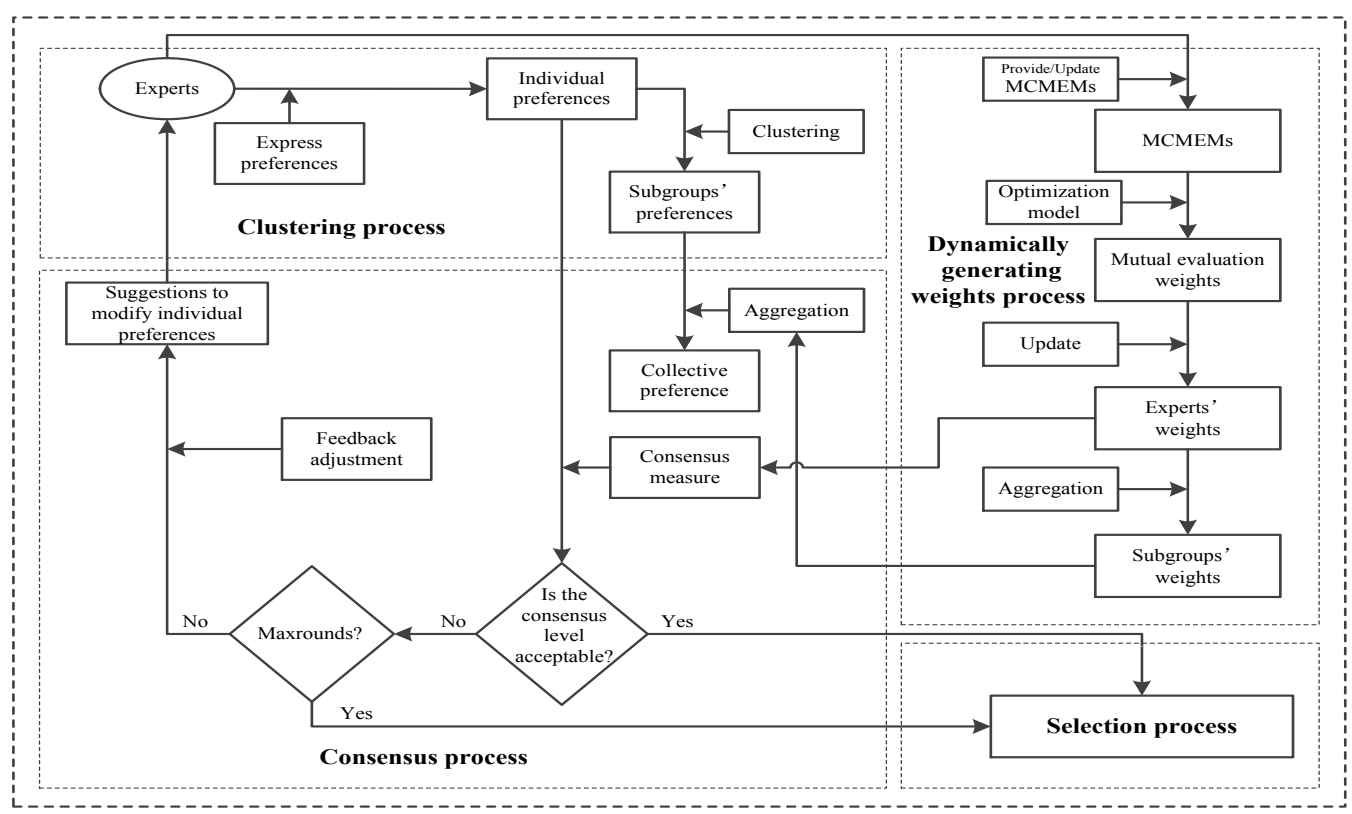

Fig.1. The large-scale consensus reaching framework

(1) Clustering process. Based on the similarity degree between experts, the Grey clustering algorithm is applied to classify experts into different subgroups.

(2) Dynamically generating weights process. In generating weights process, experts provide the MCMEMs regarding subgroups, and subgroups' weights can be obtained from the MCMEMs. Then, experts' weights can be updated dynamically by the obtained subgroups' weights. The details of the proposed dynamically generating weights process are presented in Section III-C.

(3) Consensus process. Consensus measure is used to assess the consensus level among experts, and feedback adjustment is applied to help them improve the consensus level when below a satisfactory threshold (i.e. the consensus level is unacceptable). Based on the obtained experts' dynamic weights, consensus level among experts can be computed according to Eq. (4). If the consensus level is unacceptable, the feedback adjustment is adopted to help experts modify their preference relations. Details of the consensus process have been introduced in Section II-A.

(4) Selection process. Aggregation phase and exploitation phases are carried out to obtain the ranking of alternatives. In the aggregation phase the collective preference relation is obtained via Eq. (6). Based on the obtained collective preference relation, the large group's collective preference values regarding all alternatives can be computed according to Eq. (7), from which the ranking of alternatives is derived.

\section{Dynamically generating weights process}

In the dynamically generating weights process, experts provide and update their MCMEMs regarding subgroups based on some criteria. Subgroups' weights can be obtained from the MCMEMs, and they are employed to update experts' weights.

(1) MCMEMs. We assume that there are $h$ subgroups $G=\left\{G_{1}, G_{2}, \ldots, G_{h}\right\}$ as a result of the application of the clustering process. Let $A=\left\{a_{1}, a_{2}, \ldots, a_{y}\right\} \quad(y \geq 1)$ be a set of criteria of MCMEMs, and let $\pi=\left(\pi_{1}, \pi_{2}, \ldots, \pi_{y}\right)^{T}$ be a weight vector of criteria, in which $\pi_{i} \in[0,1]$ and $\sum_{i=1}^{y} \pi_{i}=1$. Experts provide and update their MCMEMs about these subgroups using the set of criteria above in each consensus round.

Expert $e^{k}$ provides his/her MCMEMs, $V^{k}=\left(v_{i j}^{k}\right)_{h \times y}$, in which $v_{i j}^{k}$ denotes the evaluation value that expert $e_{k}$ assigns to the subgroup $G_{i}$ with respect to the criterion $a_{j}$. If $e_{k} \in G_{i}, v_{i j}^{k}=n u l l$; otherwise, $v_{i j}^{k} \in[0,100]$.

The MCMEM of subgroup $G_{t}(t=1,2, \ldots, h), V^{G_{t}}=\left(v_{i j}^{G_{t}}\right)_{h \times y}$, is obtained by averaging the provided MCMEMs of the subgroup members:

$$
v_{i j}^{G_{t}}=\sum_{e_{k} \in G_{t}} v_{i j}^{k} /\left\|G_{t}\right\|
$$

where $\left\|G_{t}\right\|$ denotes the cardinality of the subgroup. And the MCMEM of the large group, $V^{G}=\left(v_{i j}^{G}\right)_{h \times y}$, is obtained by averaging all subgroups' MCMEMs.

$$
v_{i j}^{G}=\sum_{t=1}^{h} v_{i j}^{G_{t}} /(h-1)
$$


(2) Obtaining the individuals' weights. $V^{G}$ is normalized $\overline{V^{G}}$ as follows [65]:

$$
\left\{\begin{array}{l}
\overline{v_{i j}^{G}}=v_{i j}^{G} / \sum_{i=1}^{h} v_{i j}^{G}, \text { for the benefit criterion } a_{j}, j=1,2, \ldots, y \\
\overline{v_{i j}^{G}}=\frac{1 / v_{i j}^{G}}{\sum_{i=1}^{h}\left(1 / v_{i j}^{G}\right)}, \text { for the cost criterion } a_{j}, j=1,2, \ldots, y
\end{array}\right.
$$

Let $\mu=\left(\mu^{G_{1}}, \mu^{G_{2}}, \ldots, \mu^{G_{h}}\right)^{T}$ be the weight vector of $G=\left\{G_{1}, G_{2}, \ldots, G_{h}\right\}$, where $\mu^{G_{i}} \geq 0$ is the weight of the subgroup $G_{i}$ and $\sum_{i=1}^{h} \mu^{G_{i}}=1$. The evaluation value that the large group assigns to the subgroup $G_{i}$ can be computed as $\lambda_{i}=\sum_{j=1}^{y} \pi_{j} \overline{v_{i j}^{G}}$. A large value of $\lambda_{i}$ indicates that the large group believes the subgroup $G_{i}$ is quite important. The deviation value between $\lambda_{i}$ and $\mu^{G_{i}}$ can be computed as $\left(\lambda_{i}-\mu^{G_{i}}\right)^{2}$, with the total deviation value between $\lambda_{i}$ and $\mu^{G_{i}}$ for all subgroups being $\sum_{i=1}^{h}\left(\lambda_{i}-\mu^{G_{i}}\right)^{2}$, which should be kept as small as possible. This can be assured by determining the solution $\mu=\left(\mu^{G_{1}}, \mu^{G_{2}}, \ldots, \mu^{G_{h}}\right)^{T}$ to the following nonlinear programming model:

$$
\begin{aligned}
\min & \sum_{i=1}^{h}\left(\lambda_{i}-\mu^{G_{i}}\right)^{2} \\
\text { s.t. } & \left\{\begin{array}{l}
\sum_{i=1}^{h} \mu^{G_{i}}=1 \\
\mu^{G_{i}} \geq 0, i=(1,2, \ldots, h)
\end{array}\right.
\end{aligned}
$$

By solving model (12), we can obtain the optimal solution:

$$
\mu^{G_{i}^{*}}=\sum_{j=1}^{y} \pi_{i} \overline{v_{i j}^{G}}
$$

Then, we use the obtained subgroups' weights to update the experts' weights. If the subgroup $G_{i}$ receives a large weight, weights of experts in $G_{i}$ will be increased accordingly, and vice versa. The updated weight associated to expert $e_{k} \in G_{i}, \overline{\omega_{k}}$, can thus be defined as follows:

$$
\overline{\omega_{k}}=\eta \omega_{k}+(1-\eta) \mu^{G_{i}}
$$

where $\eta$ is a parameter used to control the amount of change in updating the experts' weights, with smaller values of $\eta$ leading to a larger amount of update. In this paper, and for illustration purposes we set $\eta=0.5$.

Finally, experts' updated weights are normalized:

$$
\overline{\overline{\omega_{k}}}=\overline{\omega_{k}} / \sum_{i=1}^{m} \overline{\omega_{i}}
$$

\section{IV.Application Of The Proposed Non-COOPERATIVE BEHAVIORS MANAGEMENT FRAMEWORK}

This section introduces several common non-cooperative behaviors in large-scale GDM context, and applies the proposed large-scale consensus reaching framework to manage them. Detailed simulation experiments and comparison analysis and an illustrative example are presented to verify the validity of the proposed non-cooperative behaviors management framework.

\section{A. Non-cooperative behaviors in large-scale GDM context}

In LCRP, it is naturally that some individuals will form an alliance which may adopt non-cooperative behaviors. In this paper, we study the non-cooperative behaviors of subgroups, thus, we introduce the concept of subgroup successive similarity: for two subgroups in two successive consensus rounds, if the two subgroups share a large number of experts, then the two subgroups are considered similar and can be regarded as same. The successive similarity degree is described as follows:

Let $G_{i, z-1}$ be a subgroup in consensus round $z-1, G_{j, z}$ be a subgroup in consensus round $z$, then

$$
S D_{G_{i, z-1}, G_{j, z}}=\frac{\left\|G_{i, z-1} \mathrm{I} G_{j, z}\right\|}{\left\|G_{i, z-1} \mathrm{U} G_{j, z}\right\|}
$$

is considered as the degree of similarity between the two subgroups. The larger the value of $S D_{G_{i, z-1}, G_{j, z}}$ the higher the number of experts the two subgroups share and therefore the more similar $G_{i, z-1}$ and $G_{j, z}$ will be. In practice, in the consensus framework two subgroups in successive consensus rounds that share a large amount of experts (more than 50\%) can be considered as the same subgroup because subgroup $G_{j, z}$ will derived mainly from previous subgroup $G_{i, z-1}$. Thus, let $\chi(\chi \in[0,1])$ be a given threshold. If $S D_{G_{i, z-1}, G_{j, z}}>\chi$, the two successive consensus round subgroups are regarded the same. In this paper, as explained above we set $\chi=0.5$.

Based on the concept of subgroup similarity, we consider the following three common large-scale group non-cooperative behaviors. 


\section{1) Large-scale group non-cooperative behavior I}

In LCRP, experts' opinions will reach a consensus if they modify their preference relations according to the advice of adjustment. However, a subgroup's preference relation may diverge from the large group, and the deviation between the subgroup's preference relation and the large group's preference relation could be very large. In this paper, we call this type of behavior large-scale group non-cooperative behavior I.

Let $P^{\left(G_{i, z}\right)}$ be the preference relation of the subgroup $G_{i, z}$ in consensus round $z$, and $P^{(c, z)}$ be the large group's preference relation in that same consensus round $z$. The deviation between $P^{\left(G_{i, z}\right)}$ and $P^{(c, z)}$ can be computed as follows:

$$
N C_{1}^{G_{i, z}}=\sum_{i=1}^{n} \sum_{j=1, j \neq i}^{n}\left|p_{i j}^{\left(G_{i, z}\right)}-p_{i j}^{(c, z)}\right| /\left(n^{2}-n\right)
$$

The larger the value of $N C_{1}^{G_{i, z}}$, the larger the deviation between the subgroup $G_{i, z}$ and the large group in round $z$. Let $\alpha(\alpha \in[0,1])$ be a given threshold. If $N C_{1}^{G_{i, z}} \geq \alpha$, then we say that the subgroup $G_{i, z}$ features large-scale group non-cooperative behavior I.

2) Large-scale group non-cooperative behavior II

In the LCRP, the members of a subgroup may, to further their interests, modify their preference relations by a little or in the opposite recommended direction. In this paper, we call this type of behavior large-scale group non-cooperative behavior II.

Let $f_{i j}^{(k, z)}$ be the adjustment amount of expert $e_{k}$ regarding the pair of alternatives $\left(x_{i}, x_{j}\right)$ according to the advice of adjustment:

$$
f_{i j}^{(k, z)}= \begin{cases}\left|p_{i j}^{(k, z)}-p_{i j}^{(k, z-1)}\right|, \text { if } p_{i j}^{(k, z)} \in\left[\min \left(p_{i j}^{(k, z-1)}, p_{i j}^{(c, z-1)}\right), \max \left(p_{i j}^{(k, z-1)}, p_{i j}^{(c, z-1)}\right)\right] \\ 0, & \text { otherwise }\end{cases}
$$

Let $F^{\left(G_{i, z}\right)}$ be the total adjustment amount of experts in subgroup $G_{i, z}$, and $\overline{F^{\left(G_{i, z}\right)}}$ be the total adjustment amount of experts in subgroup $G_{i, z}$ to achieve a complete consensus:

$$
\left\{\begin{array}{l}
F^{\left(G_{i, z}\right)}=\sum_{i=1}^{n} \sum_{j=1}^{n} \sum_{e_{k} \in G_{i, z}} f_{i j}^{(k, z)} \\
F^{\left(G_{i, z}\right)}=\sum_{i=1}^{n} \sum_{j=1}^{n} \sum_{e_{k} \in G_{i, z}}\left|p_{i j}^{(k, z-1)}-p_{i j}^{(c, z-1)}\right|
\end{array}\right.
$$

To assess whether a complete consensus over all of the pairs of alternatives $\left(x_{i}, x_{j}\right)$ is achieved, we compute

$$
N C_{2}^{G_{i, z}}=1-F^{\left(G_{i, z}\right)} / \overline{F^{\left(G_{i, 2}\right)}}
$$

Clearly, the larger the value of $F^{\left(G_{i, z}\right)} / \overline{F^{\left(G_{i, z}\right)}}$, the larger adjustment proportion is implemented for subgroup $G_{i, z}$. Let $\beta(\beta \in[0,1])$ be a given threshold. If $N C_{2}^{G_{i, z}} \geq \beta$, then we say that subgroup $G_{i, z}$ features large-scale group non-cooperative behavior II.

3) Large-scale group non-cooperative behavior III

In the process of feedback adjustment, a subgroup may systematically decrease the evaluation value of the collective most preferred alternative to further its interests. In this paper, we call this type of behavior large-scale group non-cooperative behavior III.

Let $x^{(o, z-1)}$ be the collective most preferred alternative in consensus round $z-1$. Let

$$
O^{\left(G_{i, z}\right)}=\left(o^{\left(G_{i, z}\right)}\left(x_{1}\right), o^{\left(G_{i, z}\right)}\left(x_{2}\right), \ldots, o^{\left(G_{i, z}\right)}\left(x_{n}\right)\right)
$$

be the preference ordering associated with subgroup $G_{i, z}$ in consensus round $z$, where $o^{\left(G_{i, z}\right)}\left(x_{k}\right)=j$ if $x_{k}$ is the $j$ th biggest element in $U^{G_{i, z}}=\left\{U_{x_{1}}^{G_{i, z}}, U_{x_{2}}^{G_{i, z}}, \ldots, U_{x_{n}}^{G_{i, z}}\right\}$ derived from $P^{\left(G_{i, z}\right)}$ using Eq. (7). The following index, $N C_{3}^{G_{i, z}}$, is defined to check whether $G_{i, z}$ has large-scale group non-cooperative behavior III or not.

$$
N C_{3}^{G_{i, z}}= \begin{cases}1, & \text { if } o^{\left(G_{i, z}\right)}\left(x^{(o, z-1)}\right) \geq \operatorname{round}(\gamma \times n) \\ 0, & \text { otherwise }\end{cases}
$$

where $\gamma(\gamma \in[0,1])$ is a given parameter and the round is the usual rounding operation. If $N C_{3}^{G_{i, z}}=1$, then we say that subgroup $G_{i, z}$ features large-scale group non-cooperative behavior III.

Note 1: Parameters $\alpha, \beta$ and $\gamma$ are threshold values used to check whether subgroups feature large-scale group non-cooperative behaviors I-III, respectively. The larger the value of $\alpha, \beta$ and $\gamma$ the more unlikely they will reveal large-scale group non-cooperative behaviors I-III, respectively. The values of $\alpha, \beta$ and $\gamma$ can be set according to the practical decision situations to manage non-cooperative behaviors effectively. 


\section{B. Simulation experiments}

In this section, we design several simulation experiments to verify the validity of the proposed large-scale consensus reaching framework for managing non-cooperative behaviors in LCRP. The details of simulation experiments are presented below.

In the simulation experiments, we randomly generate the initial experts' preference relations. After the clustering process, experts provide their MCMEMs. The MCMEMs involve three criteria: professional skill, cooperation and fairness. In existing literature, a number of methods are proposed to set the criterion weights in multiple criteria decision making (e.g., [1, 60, 65]). In a practical large-scale GDM situation, we do not have much knowledge about which large-scale group non-cooperative behavior subgroups will adopt. In our framework, each large-scale group non-cooperative behavior is managed by one or more criteria, so we set that all criterion weights are equal to effectively manage non-cooperative behaviors. In the following, we design simulation experiments I-III based on the following natural hypotheses 1-3, respectively.

Hypothesis 1: If a subgroup features large-scale group non-cooperative behavior I, then experts in other subgroups will decrease the evaluation value of this subgroup regarding the criterion "professional skill".

Hypothesis 2: If a subgroup features large-scale group non-cooperative behavior II, then experts in other subgroups will decrease the evaluation value of this subgroup regarding the criterion "cooperation".

Hypothesis 3: If a subgroup features large-scale group non-cooperative behavior III, then experts in other subgroups will decrease the evaluation values of this subgroup regarding the criteria "fairness" and "professional skill".

Simulation experiments I-III are included in Appendices A, B and C, respectively. In order to clarify the use of Simulation experiments I-III, the following Notes are added.

Note 2: The uniform distribution and the normal distribution are widely used to generate random data in simulation experiments. The normal distribution will result in a high consensus level, and thus in Simulation experiments I-III we follow the research line of [14] and use the uniform distribution to randomly generate preference relations.

Note 3: In simulation experiments I-III, (1) the parameter $z$ denotes the iteration number to achieve a consensus; (2) the parameter $s$ reflects whether the given consensus level can be achieved or not; (3) the parameter $\theta(\theta \in[0,1])$ denotes the penalty coefficient, with a larger $\theta$ value denoting a larger penalty strength; (4) the parameter $m^{*}$ denotes the number of experts adopting large-scale group non-cooperative behaviors; (5) step 6 is used to implement preference modifications, which can guarantee that experts $e_{k} \in G_{(i, z)}$ have large-scale group non-cooperative behaviors I-III, respectively. The basic idea of step 6 is that: (a) experts $e_{k} \notin G_{(i, z)}$ modify their preferences according to the advice of adjustment (i.e. Eq. (5)) and (b) experts $e_{k} \in G_{(i, z)}$ modify their preferences satisfying the characteristics of large-scale group non-cooperative behaviors I-III defined in Section IV-A, respectively.

\section{Simulation results}

Let $z_{\max }=5$ and $\overline{c l}=0.85$. We set different input parameters: $m, n, \alpha, m^{*}$ (adopting large-scale group non-cooperative behavior I) and $\theta$ for Simulation experiment I; $m, n, \beta, m^{*}$ (adopting large-scale group non-cooperative behavior II) and $\theta$ for Simulation experiment II; and $m, n, \gamma, m^{*}$ (adopting large-scale group non-cooperative behavior III) and $\theta$ for Simulation experiment III.

We run these three simulation experiments 1000 times to obtain the average values of $s$ and $z$, which reflect the success ratio and iteration number of achieving the given consensus level in the simulation experiments, respectively. The average values of $s$ and $z$, under different input parameters for Simulation experiments I-III, are listed in Tables 1-3, respectively.

Furthermore, the average $z$ values in Simulation experiments I-III under different parameters are depicted in Figs. 2-4, respectively.

Table 1: Average values of $s$ and $z$ in Simulation experiment I under different parameters

\begin{tabular}{|c|c|c|c|c|c|c|c|c|c|c|c|c|c|c|}
\hline \multirow[b]{3}{*}{$m$} & \multirow[b]{3}{*}{$n$} & \multirow[b]{3}{*}{$\alpha$} & \multicolumn{6}{|c|}{$m^{*}=8$} & \multicolumn{6}{|c|}{$m^{*}=10$} \\
\hline & & & \multicolumn{2}{|c|}{$\theta=0.2$} & \multicolumn{2}{|c|}{$\theta=0.4$} & \multicolumn{2}{|c|}{$\theta=0.6$} & \multicolumn{2}{|c|}{$\theta=0.2$} & \multicolumn{2}{|c|}{$\theta=0.4$} & \multicolumn{2}{|c|}{$\theta=0.6$} \\
\hline & & & $s$ & $z$ & $s$ & $z$ & $s$ & $z$ & $s$ & $z$ & $s$ & $z$ & $s$ & $z$ \\
\hline \multirow[t]{4}{*}{30} & 4 & 0.20 & 0.993 & 3.660 & 1 & 3.366 & 1 & 3.069 & 0.945 & 3.913 & 0.997 & 3.554 & 1 & 3.221 \\
\hline & & 0.30 & 0.965 & 4.207 & 1 & 3.773 & 1 & 3.195 & 0.847 & 4.425 & 0.980 & 4.003 & 1 & 3.417 \\
\hline & 7 & 0.20 & 1 & 3.579 & 1 & 3.222 & 1 & 3.001 & 0.991 & 3.900 & 1 & 3.480 & 1 & 3.037 \\
\hline & & 0.30 & 0.999 & 4.078 & 1 & 3.788 & 1 & 3.011 & 0.931 & 4.442 & 1 & 4.012 & 1 & 3.212 \\
\hline \multirow[t]{4}{*}{50} & 4 & 0.20 & 1 & 3.158 & 1 & 3.032 & 1 & 3.000 & 1 & 3.300 & 1 & 3.105 & 1 & 3.001 \\
\hline & & 0.30 & 1 & 3.400 & 1 & 3.079 & 1 & 3.000 & 1 & 3.768 & 1 & 3.284 & 1 & 3.013 \\
\hline & 7 & 0.20 & 1 & 3.019 & 1 & 3.000 & 1 & 3.000 & 1 & 3.194 & 1 & 3.003 & 1 & 3.000 \\
\hline & & 0.30 & 1 & 3.148 & 1 & 3.000 & 1 & 3.000 & 1 & 3.844 & 1 & 3.047 & 1 & 3.000 \\
\hline
\end{tabular}


Table 2: Average values of $s$ and $z$ in Simulation experiment II under different parameters

\begin{tabular}{cccccccccccccccc}
\hline \multicolumn{1}{c}{$\theta$} & \multicolumn{1}{c}{$m^{*}=8$} \\
\hline & \multicolumn{1}{c}{$\theta=0.2$} & \multicolumn{1}{c}{$\theta=0.4$} & \multicolumn{1}{c}{$\theta=0.6$} & \multicolumn{1}{c}{$\theta=0.2$} & \multicolumn{3}{c}{$\theta=0.4$} & \multicolumn{2}{c}{$\theta=0.6$} \\
\hline$m$ & $n$ & $\beta$ & $s$ & $z$ & $s$ & $z$ & $s$ & $z$ & $s$ & $z$ & $s$ & $z$ & $s$ & $z$ \\
\hline 30 & 4 & 0.80 & 1 & 3.917 & 1 & 3.667 & 1 & 3.125 & 1 & 3.972 & 1 & 3.792 & 1 & 3.295 \\
& & 0.90 & 1 & 3.998 & 1 & 3.885 & 1 & 3.259 & 1 & 4.009 & 1 & 3.935 & 1 & 3.490 \\
& 7 & 0.80 & 1 & 3.992 & 1 & 3.759 & 1 & 3.007 & 1 & 4.000 & 1 & 3.907 & 1 & 3.140 \\
& & 0.90 & 1 & 4.000 & 1 & 3.979 & 1 & 3.135 & 1 & 4.000 & 1 & 3.998 & 1 & 3.520 \\
50 & 4 & 0.80 & 1 & 3.406 & 1 & 3.048 & 1 & 3.002 & 1 & 3.800 & 1 & 3.227 & 1 & 3.007 \\
& & 0.90 & 1 & 3.812 & 1 & 3.188 & 1 & 3.002 & 1 & 3.975 & 1 & 3.598 & 1 & 3.017 \\
& 7 & 0.80 & 1 & 3.369 & 1 & 3.000 & 1 & 3.000 & 1 & 3.933 & 1 & 3.066 & 1 & 3.000 \\
& & 0.90 & 1 & 3.943 & 1 & 3.056 & 1 & 3.000 & 1 & 4.000 & 1 & 3.660 & 1 & 3.000 \\
\hline
\end{tabular}

Table 3: Average values of $s$ and $z$ in Simulation experiment III under different parameters

\begin{tabular}{|c|c|c|c|c|c|c|c|c|c|c|c|c|c|c|}
\hline \multirow[b]{3}{*}{$m$} & \multirow[b]{3}{*}{$n$} & \multirow[b]{3}{*}{$\gamma$} & \multicolumn{6}{|c|}{$m^{*}=8$} & \multicolumn{6}{|c|}{$m^{*}=10$} \\
\hline & & & \multicolumn{2}{|c|}{$\theta=0.2$} & \multicolumn{2}{|c|}{$\theta=0.4$} & \multicolumn{2}{|c|}{$\theta=0.6$} & \multicolumn{2}{|c|}{$\theta=0.2$} & \multicolumn{2}{|c|}{$\theta=0.4$} & \multicolumn{2}{|c|}{$\theta=0.6$} \\
\hline & & & $s$ & $z$ & $s$ & $z$ & $s$ & $z$ & $s$ & $z$ & $s$ & $z$ & $s$ & $z$ \\
\hline \multirow[t]{4}{*}{30} & 4 & 0.50 & 0.994 & 3.711 & 1 & 3.412 & 1 & 3.160 & 0.943 & 3.919 & 0.996 & 3.632 & 1 & 3.275 \\
\hline & & 0.80 & 0.992 & 3.724 & 1 & 3.428 & 1 & 3.177 & 0.931 & 3.947 & 0.994 & 3.641 & 1 & 3.300 \\
\hline & 7 & 0.50 & 1 & 3.640 & 1 & 3.265 & 1 & 3.024 & 0.987 & 3.967 & 1 & 3.558 & 1 & 3.092 \\
\hline & & 0.80 & 1 & 3.657 & 1 & 3.273 & 1 & 3.037 & 0.985 & 4.029 & 1 & 3.563 & 1 & 3.094 \\
\hline \multirow[t]{4}{*}{50} & 4 & 0.50 & 1 & 3.176 & 1 & 3.059 & 1 & 3.043 & 1.000 & 3.351 & 1 & 3.157 & 1 & 3.064 \\
\hline & & 0.80 & 1 & 3.185 & 1 & 3.074 & 1 & 3.052 & 1.000 & 3.357 & 1 & 3.167 & 1 & 3.080 \\
\hline & 7 & 0.50 & 1 & 3.024 & 1 & 3.003 & 1 & 3.006 & 1.000 & 3.202 & 1 & 3.025 & 1 & 3.016 \\
\hline & & 0.80 & 1 & 3.027 & 1 & 3.009 & 1 & 3.007 & 1.000 & 3.218 & 1 & 3.027 & 1 & 3.019 \\
\hline
\end{tabular}

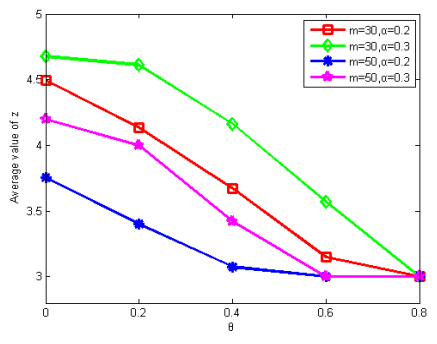

(a) $n=7, m^{*}=12$

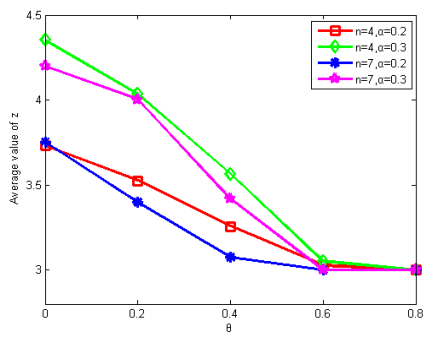

(b) $m=50, m^{*}=12$

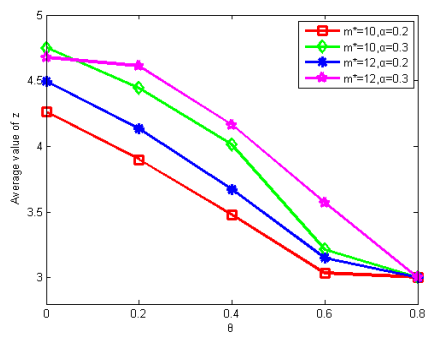

(c) $m=30, n=7$

Fig. 2. Average $z$ values in Simulation experiment I under different parameters
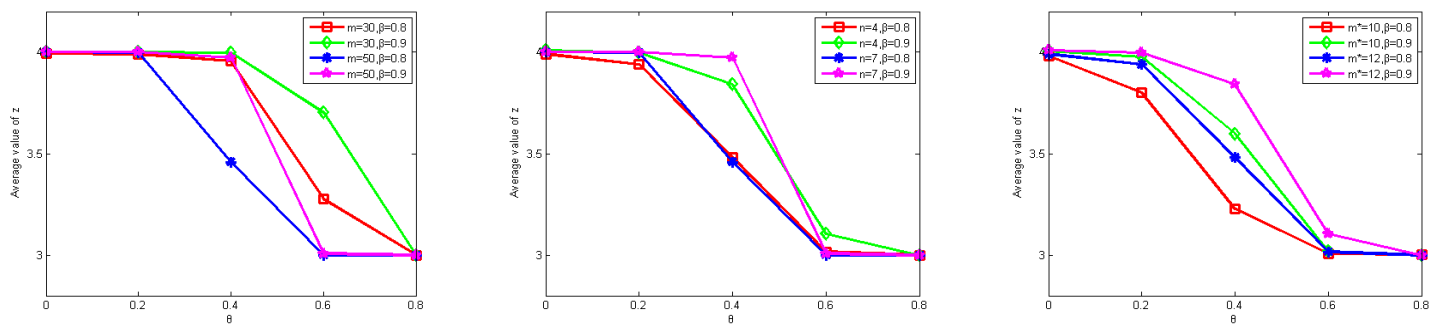

(a) $n=7, m^{*}=12$

(b) $m=50, m^{*}=12$

(c) $m=50, n=4$

Fig. 3. Average $z$ values in Simulation experiment II under different parameters

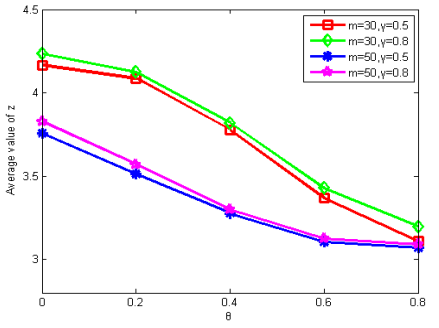

(a) $n=4, m^{*}=12$

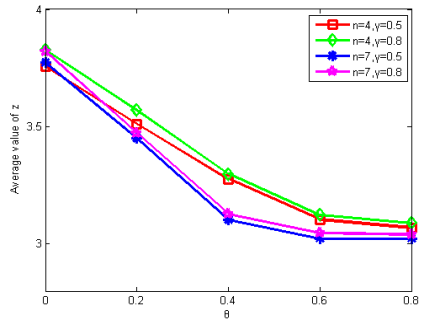

(b) $m=50, m^{*}=12$

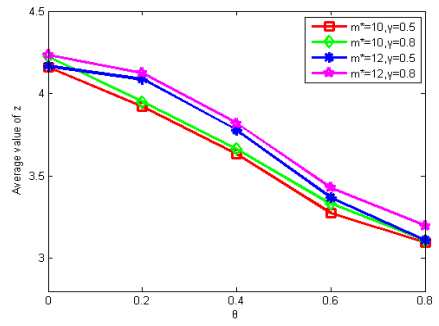

(c) $m=30, n=4$

Fig. 4. Average $z$ values in Simulation experiment III under different parameters 
From Tables 1-3 and Figs. 2-4, we can draw the following observations:

(1) The proposed large-scale consensus reaching framework can manage large-scale group non-cooperative behaviors I-III effectively when setting different parameters. Generally, it needs an average of 3-4 rounds to achieve a consensus, and it has high consensus success ratios (close to 1 ).

(2) When the proportion of the experts who adopt non-cooperative behaviors increases, the ability of the proposed consensus reaching framework for managing non-cooperative behaviors decreases.

(3) When decreasing the values of $\alpha, \beta$ and $\gamma$ or increasing the values of $\theta$, the average values of $z$ decreases, while the average values of $s$ increase, which means that adopting a relaxed criteria to detect the non-cooperative behaviors or using a strong penalty will accelerate the speed to achieve a consensus and will improve the success ratio of achieving a consensus.

\section{Comparative analysis}

In this section, we compare the proposed large-scale group non-cooperative behaviors management framework against the traditional LCRP to verify the validity of our framework.

In traditional CRPs, experts' weights remain unchanged. The traditional methods can be also exported to manage large-scale GDM after some minor modifications. In our simulation experiments, when deleting the dynamically generating weights process, we obtain the traditional LCRP. In Simulation experiments I-III, we remove steps 3-5 to obtain Simulation experiments I*-III ${ }^{*}$, which can be used to describe traditional LCRP.

Let $n=6, z_{\max }=5, \overline{c l}=0.9, \theta=0.2$, and $m^{*}=10$. When setting different input parameters $m$ and $\alpha$ for simulation methods $\mathrm{I}^{*}$ and $\mathrm{I}$, we run these two simulation experiments 1000 times, obtaining the average values of $s$ and $z$. The simulation results are described in Fig. 5.

Let $n=6, z_{\max }=5, \overline{c l}=0.9, \theta=0.2$, and $m^{*}=10$. When setting different input parameters $m$ and $\beta$ for simulation methods II* and II, we run these two simulation experiments 1000 times, obtaining the average values of $s$ and $z$. The simulation results are described in Fig. 6.

Let $n=8, z_{\max }=5, \overline{c l}=0.9, \theta=0.2$, and $m^{*}=10$. When setting different input parameters $m$ and $\gamma$ for simulation methods III* and III, we run these two simulation experiments 1000 times, obtaining the average values of $s$ and $z$. The simulation results are presented in Fig. 7.
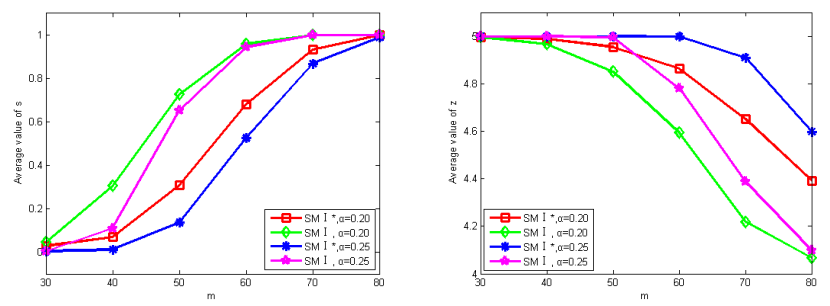

Fig. 5. Average $s$ and $z$ values in Simulation experiments I and I* under different parameters $m$ and $\alpha$
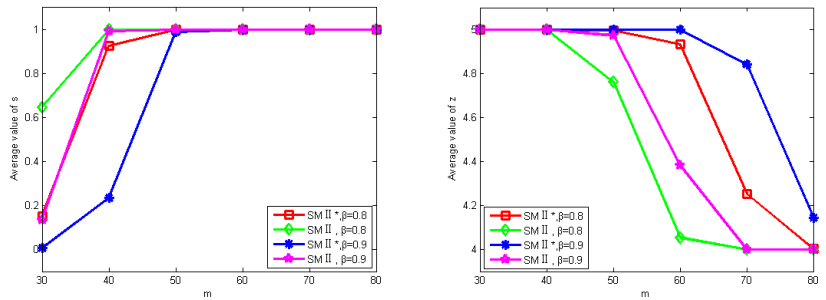

Fig. 6. Average $s$ and $z$ values in Simulation experiments II and II* under different parameters $m$ and $\beta$
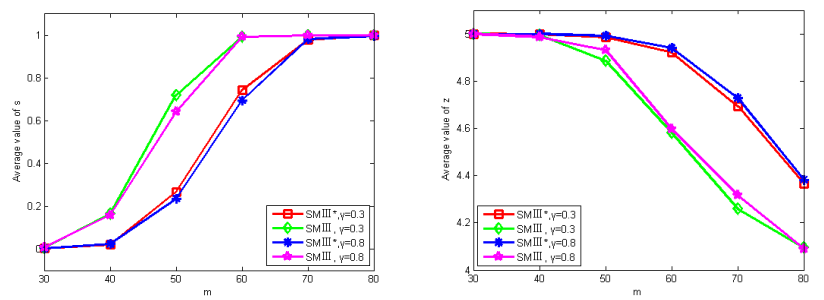

Fig. 7. Average $s$ and $z$ values in Simulation experiments III and III* under different parameters $m$ and $\gamma$ 
From Figs. 5-7, we draw the following observations:

(1) There are clearly fewer average consensus rounds in the proposed large-scale group non-cooperative behaviors management framework than those in the traditional LCRP, which means that the proposed framework can accelerate the speed to achieve a consensus.

(2) The consensus success ratios in the proposed large-scale group non-cooperative behaviors management framework are obviously higher than those in the traditional LCRP, which means that the proposed framework can improve the success ratio of achieving a consensus by managing the non-cooperative behaviors.

\section{E. Illustrative example}

In this section, we present an illustrative example to show how the consensus level and experts' weights change in five successive rounds in the traditional LCRP and in the proposed self-management mechanism-based large-scale consensus reaching framework. In this example, we assume that there are 30 experts, $E=\left\{e_{1}, e_{2}, \ldots, e_{30}\right\}$. They provide their preference relations, $P=\left\{P^{1}, P^{2}, \ldots, P^{30}\right\}$, regarding a set of 6 alternatives $X=\left\{x_{1}, x_{2}, \ldots, x_{6}\right\}$. Meanwhile, we also assume that there are 10 experts, $E^{*}=\left\{e_{21}, e_{22}, \ldots, e_{30}\right\}$, who have non-cooperative behaviors. Let $E \backslash E^{*}$ be the experts without non-cooperative behaviors.

In the following, we use the traditional LCRP and the self-management mechanism-based consensus reaching framework to help the 30 experts to achieve a consensus. Because of the limited space, we omit the original and adjusted preference relations, and MCMEMs in the LCRP. Besides, we only consider large-scale group non-cooperative behavior I in this example. If we consider large-scale group non-cooperative behaviors II or III, we obtain similar results.

In the self-management mechanism-based consensus reaching framework, we set $\alpha=0.25$ to reveal large-scale group non-cooperative behavior I, and use $\theta=0.5$ to penalize the subgroup with large-scale group non-cooperative behavior I. We can respectively obtain the consensus level and the average weight of $E^{*}$ and the average weight of $E \backslash E^{*}$ in the traditional LCRP and in the proposed self-management mechanism-based framework in five successive rounds as Fig. 8 illustrates.
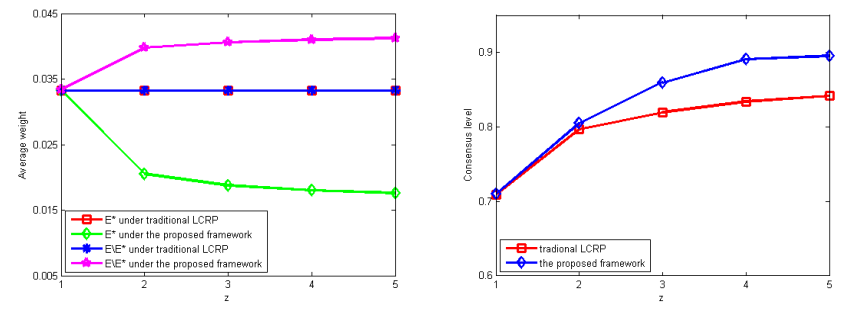

Fig.8. The consensus level and the average weight of experts under traditional LCRP and our proposal

From Figs. 8, we observe that in the self-management mechanism-based large-scale consensus reaching framework the average weight of experts with non-cooperative behaviors are obviously decreased in contrast with the traditional LCRP where they remain unchanged. Furthermore, the speed to achieve a consensus under the proposed large-scale consensus reaching framework is faster than that in the traditional LCRP. These findings imply that the proposed framework can manage non-cooperative behaviors effectively in the LCRP.

\section{DISCUSSION: ADVANTAGES AND LIMITATIONS}

In this section, we point out some advantages and limitations of the proposed self-management mechanism to manage non-cooperative behaviors in the LCRP.

(1) Advantages: We find the following advantages of our proposal.

(a) Compared with Pelta and Yager [42], Yager [62,63], and Dong et al. [10], non-cooperative behaviors are considered not only in the aggregation process but also in the CRP of GDM.

(b) In the works of Palomares et al. [41] and Quesada et al. [44], the management of the non-cooperative behaviors is heavily dependent on a moderator and is occasionally excessively demanding for the moderator. Compared with [41, 44], the mechanism presented in this paper does not rely on the moderator, and it depends on the mutual evaluations among subgroups.

(c) The mechanism presented in Dong et al. [14] is suitable for decision contexts that involve a small number of experts, but not for large-scale GDM contexts because of the difficulty to manage mutual evaluation matrices among a large number of experts. The proposed model in this paper is designed for large-scale GDM contexts to overcome this specific shortcoming of Dong et al.'s model [14].

(d) Detailed simulation experiments are designed and reported to verify the validity of the proposed model for managing non-cooperative behaviors in the LCRP. The simulation results show that our model can manage large-scale group non-cooperative behaviors effectively.

(2) Limitations: We find the following limitations, which require of future research efforts in their addressing.

(a) In existing non-cooperative behavior studies in CRPs, simulation experiments have been used to show the effectiveness of the proposed models $[14,41,44,61]$. The simulation method is also adopted in this study. Inevitably, we make some basic 
assumptions and set several parameters values in the running of the simulation experiments. Thus, simulation experiments are based on some assumptions and parameters setting, and do not cover all possible actual decision situations in real-world LCRPs, which are more complex than those considered in simulation experiments. It could be interesting in future research to investigate non-cooperative behaviors in the LCRP empirically.

(b) In this paper, we discuss the self-management mechanism to manage non-cooperative behaviors in the LCRP by analyzing whether a consensual collective solution can be achieved. We argue that it will be interesting to investigate the non-biased outcomes in the proposed LCRP framework based on the latest research results on CRP (e.g., Gołuńska and Hołda [20], and Kacprzyk and Zadrożny [30]).

(c) To our knowledge, there is a lack of theoretical analysis of non-cooperative behaviors in CRPs in the existing literature because the challenge posed of CRPs within a complex and dynamic process, although it is arguably a necessity to get theoretical analysis regarding non-cooperative behaviors in the LCRP..

\section{CONCLUSION}

In LCRP non-cooperative behaviors happen. In this paper we proposed a novel framework based on a self-management mechanism to manage large-scale group non-cooperative behaviors. In this framework, experts not only provide preference relations, but also provide and update MCMEMs based on subgroups' performance from which subgroups' weights are obtained. Then, individuals' weights can be dynamically updated by the obtained subgroups' weights, and they are integrated into the LCRP. We consider several common large-scale group non-cooperative behaviors in LCRP. If a subgroup is detected to use large-scale group non-cooperative behaviors, experts in other subgroups will decrease the evaluation values of this subgroup which eventually will decrease the weights of experts in this subgroup. We designed detailed simulation experiments and comparison analysis to verify the validity of the proposed framework for managing these non-cooperative behaviors in LCRP.

In real-world, decision elements can be dynamically changed during the LCRP, examples of which are the experts' participation rate, or the number and type of available alternatives [43]. Meanwhile, social relationships (such as social network $[16,33,54])$ among experts may play a key role in the consensus building. We believe that it will be very interesting in future to investigate and deal with the dynamic elements and social relations issues in the self-management mechanism-based framework in the LCRP.

\section{APPENDIX A. SIMULATION EXPERIMENT I}

Input: $m, n, m^{*}, \overline{c l}, \mathrm{z}_{\max }, \theta$ and $\alpha$.

Output: $s, z$.

Step 1: Let $z=1$. Initialize experts' preference relations and weights. We randomly generate $m n \times n$ preference relations. We set that $m^{*}$ experts, $\bar{G}=\left\{e_{\Delta(1)}, e_{\Delta(2)}, \ldots, e_{\Delta\left(m^{*}\right)}\right\}$, have non-cooperative behavior I.

Step 2: Clustering. Compute similarity degree $s^{k l}(k=1, \ldots, m-1, l=k+1, \ldots, m)$ between experts $e^{k}$ and $e^{l}$ by Eq. (8), and then classify experts into a number of subgroups $\left\{G_{1, z}, G_{2, z}, \ldots, G_{h, z}\right\}$ by the Grey clustering algorithm.

Step 3: Obtaining MCMEMs. If $z=1$, for $e_{k} \in G_{t}(t=1,2,,, h), v_{t j}^{(k, z)}=n u l l$; for $e_{k} \notin G_{t, z}$, generate $v_{t j}^{(k, z)}$ randomly from interval [80,100]. If $z \geq 2$, according to hypothesis $\mathbf{1}$, experts in other subgroups will decrease the evaluation values of the subgroup $\bar{G}$ regarding the criterion "professional skill". The process is presented as follows:

$$
\begin{cases}v_{i j}^{(k, z)}=\text { null }, & e_{k} \in G_{i, z} \\ v_{i j}^{(k, z)} \in[80,100], & e_{k} \notin G_{i, z} \& G_{i, z} \neq \bar{G} \\ v_{i j}^{(k, z)} \in[80,100], & e_{k} \notin G_{i, z} \& G_{i, z}=\bar{G} \& j=2,3 \\ v_{i j}^{(k, z)} \in[\max (80-100 \theta, 0), 100-100 \theta], & e_{k} \notin G_{i, z} \& G_{i, z}=\bar{G} \& j=1\end{cases}
$$

Step 4: Obtain experts' weights and collective preference relation. Based on obtained MCMEMs, use Eqs. (9)-(13) to obtain subgroups' weights, $\mu=\left(\mu^{G_{1, z}}, \mu^{G_{2, z}}, \ldots, \mu^{G_{h, z}}\right)$. Then use the obtained subgroups weights to update experts' initial weights by Eq. (14) and obtain collective preference relation by Eq. (6).

Step 5: Consensus measure. Use Eq. (4) to obtain the consensus level $c l$ among experts. If $c l \geq \overline{c l}$ or $z \geq z_{\max }$, then go to step 7; otherwise, continue with the next step.

Step 6: Feedback adjustment. For $e_{k} \notin \bar{G}$, Eq. (5) is used to modify their preference relations, and guarantee that they do not have non-cooperative behavior I; for $e_{k} \in \bar{G}$, generate experts' preference relations, and guarantee that the deviation between the subgroup $\bar{G}$ and the large group is larger than $\alpha$.

Let $z=z+1$, then go to step 2 .

Step 7: Output. If $c l \geq \overline{c l}$, then $s=1$; otherwise, $s=0$. Output $z$ and $s$. 


\section{APPENDIX B. SIMULATION EXPERIMENT II}

Output, Step 2, Step 4, Step 5 and Step 7 are the same as simulation experiment I, we omit them.

Input: $m, n, m^{*}, \overline{c l}, \mathrm{z}_{\max }, \theta$ and $\beta$.

Step 1: Let $z=1$. Initialize experts' preference relations and weights. We randomly generate $m n \times n$ preference relations. We set that $m^{*}$ experts, $\bar{G}=\left\{e_{\Delta(1)}, e_{\Delta(2)}, \ldots, e_{\Delta\left(m^{*}\right)}\right\}$, have non-cooperative behavior II.

Step 3: Obtaining MCMEMs. If $z=1$, for $e_{k} \in G_{t}(t=1,2,,, h), v_{t j}^{(k, z)}=n u l l$; for $e_{k} \notin G_{t, z}$, generate $v_{t j}^{(k, z)}$ randomly from interval [80,100]. If $z \geq 2$, according to hypothesis 2 , experts in other subgroups will decrease the evaluation values of the subgroup $\bar{G}$ regarding the criterion "cooperation". The process is presented as follows:

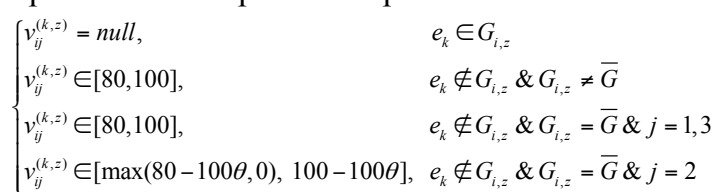

Step 6: Feedback adjustment. For $e^{k} \notin \bar{G}$, Eq. (5) is used to modify their preference relations, and guarantee that they do not have non-cooperative behavior II; for $e^{k} \in \bar{G}$, use following rule to modify their preference relations.

Let $p_{i j}^{(k, z+1)}=p_{i j}^{(c, z)} \times \beta \times \tau+p_{i j}^{(k, z)} \times(1-\beta \times \tau)$, where $\tau \in[0,1]$ is a random value. Let $z=z+1$, then go to step 2 .

\section{APPENDIX C. SIMULATION EXPERIMENT III}

Output, Step 2, Step 4, Step 5 and Step 7 are the same as simulation experiment I, we omit them.

Input: $m, n, m^{*}, \overline{c l}, \mathrm{z}_{\max }, \theta$ and $\gamma$.

Step 1: Let $z=1$. Initialize experts' preference relations and weights. We randomly generate $m n \times n$ preference relations. We set that $m^{*}$ experts, $\bar{G}=\left\{e_{\Delta(1)}, e_{\Delta(2)}, \ldots, e_{\Delta\left(m^{*}\right)}\right\}$, have non-cooperative behavior III.

Step 3: Obtaining MCMEMs. If $z=1$, for $e_{k} \in G_{t}(t=1,2,,, h), v_{t j}^{(k, z)}=n u l l$; for $e_{k} \notin G_{t, z}$, generate $v_{t j}^{(k, z)}$ randomly from interval [80,100]. If $z \geq 2$, according to hypothesis 3 , experts in other subgroups will decrease the evaluation values of the subgroup $\bar{G}$ regarding the criteria "professional skill" and "fairness". The process is presented as follows:

$$
\begin{cases}v_{i, k}^{(k, z)}=\text { null, } & e_{k} \in G_{i, z} \\ v_{i, j}^{(k, z)} \in[80,100], & e_{k} \notin G_{i, z} \& G_{i, z} \neq \bar{G} \\ v_{i, k}^{(k, z)} \in[80,100], & e_{k} \notin G_{i, z} \& G_{i, z}=\bar{G} \& j=2 \\ v_{v_{i j}(k, z)} \in[\max (80-100 \theta, 0), 100-100 \theta], & e_{k} \notin G_{i, z} \& G_{i, z}=\bar{G} \& j=1,3\end{cases}
$$

Step 6: Feedback adjustment. For $e_{k} \notin \bar{G}$, Eq. (5) is used to modify their preference relations according, and guarantee that they do not have non-cooperative behavior III; for $e_{k} \in \bar{G}$, use following rule to modify their preference relations.

Let $x^{(o, z)}$ be the collective most preferred alternative in round $z, O^{(\bar{G}, z)}=\left(o^{(\bar{G}, z)}\left(x_{1}\right), o^{(\bar{G}, z)}\left(x_{2}\right), \ldots, o^{(\bar{G}, z)}\left(x_{n}\right)\right)$ be the preference ordering associated with the subgroup $\bar{G}$ in round $z$, where $o^{(\bar{G}, z)}\left(x_{i}\right)=j$ if $x_{i}$ is the jth biggest element in $U^{\bar{G}, z}=\left\{U_{x_{1}}^{\bar{G}, z}, U_{x_{2}}^{\bar{G}, z}, \ldots, U_{x_{n}}^{\bar{G}, z}\right\}$, and $U^{\bar{G}, z}$ can be obtained according to $P^{(\bar{G}, z)}$ using Eq. (7). Guarantee that $o^{(\bar{G}, z+1)}\left(x^{(o, z)}\right) \geq \operatorname{round}(\gamma \times n)$. Let $z=z+1$, then go to step 2.

\section{REFERENCES}

[1] F.H. Barron, B.E. Barrett, Decision quality using ranked attribute weights, Management Science 42 (1996) 1515-1523.

[2] A. Baraldi, P. Blonda, A survey of fuzzy clustering algorithms for pattern recognition, IEEE Transactions on Systems, Man, and Cybernetics Part B: Cybernetics 29 (1999) 778-785.

[3] D. Ben-Arieh, T. Easton, Multi-criteria group consensus under linear cost opinion elasticity, Decision Support Systems 43 (2007) $713-721$.

[4] D. Ben-Arieh, T. Easton, B. Evans, Minimum cost consensus with quadratic cost functions, IEEE Transactions on Systems, Man, and Cybernetics Part A: Systems and Humans 39 (2008) 210-217.

[5] G. Bordogna, M. Fedrizzi, G. Pasi, A linguistic modeling of consensus in group decision making based on OWA operators, IEEE Transactions on Systems, Man and Cybernetics, Part A: Systems and Humans 27 (1997) 126-133.

[6] F. J. Cabrerizo, J.M. Moreno, I.J. Pérez, E. Herrera-Viedma, Analyzing consensus approaches in fuzzy group decision making: advantages and drawbacks, Soft Computing 14 (2010) 451-463.

[7] X. Chen, H.J. Zhang, Y.C. Dong, The fusion process with heterogeneous preference structures in group decision making: A survey, Information Fusion 24 (2015) 72-83. 
[8] F. Chiclana, J.M. Tapia García, M.J. del Moral, E. Herrera-Videdma, A statistical comparative study of different similarity measures of consensus in group decision making, Information Sciences 221 (2013) 110-123.

[9] A.K. Choudhury, R. Shankar, M.K. Tiwari, Consensus-based intelligent group decision-making model for the selection of advanced technology, Decision Support Systems 42 (2006) 1776-1799.

[10] Y.C. Dong, Y.T. Liu, H.M. Liang, F. Chiclana, E. Herrera-Viedma, Strategic weight manipulation in multiple attribute decision making, Omega 75 (2018) $154-164$.

[11] Y.C. Dong, C.C. Li, Y.F. Xu, X. Gu, Consensus-based group decision making under multi-granular unbalanced 2-tuple linguistic preference relations, Group Decision and Negotiation 24 (2015) 217-242.

[12] Y.C. Dong, X. Chen, F. Herrera, Minimizing adjusted simple terms in the consensus reaching process with hesitant linguistic assessments in group decision making, Information Sciences 297 (2015) 95-117.

[13] Y.C. Dong, Z. G. Ding, L. Martínez, F. Herrera, Managing consensus based on leadership in opinion dynamics, Information Sciences 397-398 (2017) 187-205.

[14] Y.C. Dong, H.J. Zhang, E. Herrera-Viedma, Integrating experts' weights generated dynamically into the consensus reaching process and its application in managing non-cooperative behaviors, Decision Support Systems (84) 2016 1-15.

[15] Y.C. Dong, H.J. Zhang, E. Herrera-Viedma, Consensus reaching model in the complex and dynamic MAGDM problem, Knowledge-Based Systems 106 (2016) 206-219.

[16] R. Efremov, D. Rios-Insua, A. Lotov, A framework for participatory decision support using pareto frontier visualization, goal identification and arbitration, European Journal of Operational Research 199 (2009) 459-467.

[17] M.T. Escobar, J. Aguarón, J.M. Moreno-Jiménez, Some extensions of the precise consistency consensus matrix, Decision Support Systems 74 (2015) 67-77.

[18] F. Franceschini, D. Maisano, Checking the consistency of the solution in ordinal semi-democratic decision-making problems, Omega 57 (2015) $188-195$.

[19] M. Fedrizzi, J. Kacprzyk, S. Zadrożny, An interactive multi-user decision support system for consensus reaching processes using fuzzy logic with linguistic quantifiers, Decision Support Systems 4 (1988) 313-327.

[20] D. Gołuńska, M. Hołda, The need of fairness in group consensus reaching process in a fuzzy environment, Czasopismo Techniczne, Automatyka 1-AC (2013) 29-38.

[21] Z.W. Gong, X.X. Xu, L.S. Xu, Consensus modeling with nonlinear utility and cost constraints: A case study, Knowledge-Based Systems 88 (2015) 210-222.

[22] Z.W. Gong, H.H. Zhang, J. Forrest, L.S. Li, X.X. Xu, Two consensus models based on the minimum cost and maximum return regarding either all individuals or one individual, European Journal of Operational Research 240 (2015) 183-192.

[23] T. González-Arteaga, R. De Andrés Calle, F. Chiclana, A new measure of consensus with fuzzy preference relations: The correlation consensus degree, Knowledge-Based Systems 107 (2016) 104-116.

[24] E. Herrera-Viedma, F.J. Cabrerizo, J. Kacprzyk, W. Pedrycz, A review of soft consensus models in a fuzzy environment, Information Fusion 17 (2014) 4-13.

[25] E. Herrera-Viedma, F. Chiclana, F. Herrera, S. Alonso, Group decision-making model with incomplete fuzzy preference relations based on additive consistency, IEEE Transactions on Systems, Man, and Cybernetics, Part B: Cybernetics 31 (2007) 227-234.

[26] E. Herrera-Viedma, F. Herrera, F. Chiclana, A consensus model for multiperson decision making with different preference structures, IEEE Transactions on Systems, Man and Cybernetics Part A: Systems and Humans 32 (2002) 394-402.

[27] J. Kacprzyk, Group decision making with a fuzzy linguistic majority, Fuzzy Sets and Systems 18 (1986) 105-118.

[28] J. Kacprzyk, M. Fedrizzi, A 'soft' measure of consensus in the setting of partial (fuzzy) preferences, European Journal of Operational Research 34 (1988) 316-325.

[29] J. Kacprzyk, M. Fedrizzi , H. Nurmi, Soft degrees of consensus under additive preferences and fuzzy majorities, in J. Kacprzyk, H. Nurmi, M. Fedrizzi (Eds.): Consensus under Fuzziness, Kluwer, Boston (1996) 55- 83.

[30] J. Kacprzyk, S. Zadrożny, On a fairness type approach to consensus reaching support under fuzziness via linguistic summaries, IEEE International Conference on Fuzzy Systems (FUZZ-IEEE) (2016) 1999-2006.

[31] J. Kacprzyk, S. Zadrozny, Soft computing and Web intelligence for supporting consensus reaching, Soft Computing 14 (2010) 833-846.

[32] G. Klir, B. Yuan, Fuzzy Sets and Fuzzy Logic: Theory and Applications, Englewood Cliffs 1995.

[33] J. Kim, A model and case for supporting participatory public decision making in e-democracy, Group Decision and Negotiation 17 (2008) $179-193$.

[34] S.F. Liu, Y.G. Dang, Z.G. Fang, et al., Grey system theory and application, Beijing: Science Press 2010.

[35] J. MacQueen, Some methods for classification and analysis of multivariate observation, Proc. 5th Berkeley Symp (1967) 281-297.

[36] S.A. Orlovsky, Decision-making with a fuzzy preference relation, Fuzzy Sets and Systems (1978) 155-167.

[37] I. Palomares, Consensus model for large-scale group decision support in IT services management, Intelligent Decision Technologies 8 (2014) 81-94.

[38] I. Palomares, F. J. Estrella, L. Martínez, F. Herrera, Consensus under a fuzzy context: Taxonomy, analysis framework AFRYCA and experimental case of study, Information Fusion 20 (2014) 252-271.

[39] I. Palomares, J. Liu, Y. Xu, L. Martínez, Modelling experts' attitudes in group decision making, Soft Computing 16 (2012) $1755-1766$.

[40] I. Palomares, L. Martínez, A semisupervised multiagent system model to support consensus-reaching processes, IEEE Transactions on Fuzzy Systems 22(2014) 762-777.

[41] I. Palomares, L. Martínez, F. Herrera, A consensus model to detect and manage noncooperative behaviors in large-scale group decision making, IEEE Transactions on Fuzzy Systems 22 (2014) 516-530.

[42] D.A. Pelta, R.R. Yager, Decision strategies in mediated multiagent negotiations: An optimization approach, IEEE Transactions on Systems, Man and Cybernetics Part A: Systems and Humans 40 (2010) 635-640.

[43] I.J. Pérez, F.J. Cabrerizo, E. Herrera-Viedma, A mobile decision support system for dynamic group decision-making problems, IEEE Transactions on Systems, Man, and Cybernetics Part A: Systems and Humans 40 (2010) 1244-1256.

[44] F.J. Quesada, I. Palomares, L.Martínez, Managing experts behaviors in large-scale consensus reaching process with uninorm aggregation operators, Applied Soft Computing 35 (2015) 873-887.

[45] R.M. Rodríguez, I. Palomares, L.Martínez, Attitude-based consensus model for heterogeneous group decision making, F. Sun et al. (Eds.), Knowledge Engineering and Management, Advances in Intelligent Systems and Computing 214, Springer 2014.

[46] T. L. Saaty, The analytic hierarchy process, McGraw-Hill, New York 1980.

[47] F. Seo, M, Sakawa, Fuzzy multiattribute utility analysis for collective choice, IEEE Transactions on Systems, Man and Cybernetics 15 (1985) 45-53.

[48] B. Srdjevic, Linking analytic hierarchy process and social choice methods to support group decision-making in water management, Decision Support Systems 42 (2007) 2261-2273.

[49] B. Srdjevic, Z. Srdjevic, B. Blagojevic, K. Suvocarev, A two-phase algorithm for consensus building in AHP-group decision making, Applied Mathematical Modelling 37 (2013) 6670-6682.

[50] B.Z. Sun, W.M. Ma, An approach to consensus measurement of linguistic preference relations in multi-attribute group decision making and application, Omega 51 (2015) 83-92.

[51] T. Tanino, On group decision making under fuzzy preferences, in: J. Kacprzyk, M. Fedrizzi (Eds.), Multiperson decision making using fuzzy sets and possibility theory, Kluwer Academic Publishers, Dordrecht (1990) 172-185. 
[52] M. Tavana, D.T. Kennedy, P. Joglekar, A group decision support framework for consensus ranking of technical manager candidates, Omega 24 (1996) 523-538.

[53] T. Wu, X.W. Liu, An interval type-2 clustering solution for large-scale multiple-criteria group decision making problems, Knowledge-Based Systems 114 (2016) 118-127.

[54] T. Wu, X.W. Liu, F. Liu, An interval type-2 fuzzy TOPSIS model for large scale group decision making problems with social network information, Information Sciences 432 (2018) 392-410.

[55] T. Wu, X.W. Liu, J. Qin, A linguistic solution for double large-scale group decision-making in E-commerce, Computers \& Industrial Engineering 116 (2018) 97-112.

[56] Z.B. Wu, J.P. Xu, A consistency and consensus based decision support model for group decision making with multiplicative preference relations, Decision Support Systems 52 (2012) 757-767.

[57] Z.B. Wu, J.P. Xu, Managing consistency and consensus in group decision making with hesitant fuzzy linguistic, Omega 65 (2016) $28-40$.

[58] J. Wu, F. Chiclana, A social network analysis trust-consensus based approach to group decision-making problems with interval-valued fuzzy reciprocal preference relations, Knowledge-Based Systems 59 (2014) 97-107.

[59] J. Wu, F. Chiclana, Multiplicative consistency of intuitionistic reciprocal preference relations and its application to missing values estimation and consensus building, Knowledge-Based Systems 71 (2014) 187-200.

[60] J.P. Xu, Z.B. W, A discrete consensus support model for multiple attribute group decision making, Knowledge-Based Systems 24 (2011) $1196-1202$.

[61] X.H. Xu, Z.J. Du, X.H. Chen, Consensus model for multi-criteria large-group emergency decision making considering non-cooperative behaviors and minority opinions, Decision Support Systems 79 (2015) 150-160.

[62] R.R. Yager, Penalizing strategic preference manipulation in multi-agent decision making, IEEE Transactions on Fuzzy Systems 9 (2001) 393-403.

[63] R.R. Yager, Defending against strategic manipulation in uninorm-based multi-agent decision making, European Journal of Operational Research 141 (2002) 217-232.

[64] R.R. Yager, Quantifier guided aggregation using OWA operators, International Journal of Intelligent Systems 11 (1996) 49-73.

[65] K. Yoon, C.L. Hwang, Multiple attribute decision making: methods and applications, Springer, Berlin 1981.

[66] H.J. Zhang, Y.C. Dong, X. Chen, The 2-Rank consensus reaching model in the multigranular linguistic multiple-attribute group decision-making, IEEE Transactions on Systems, Man, and Cybernetics. (in press, doi: 10.1109/TSMC.2017.2694429).

[67] H.J. Zhang, Y.C. Dong, E. Herrera-Viedma, Consensus building for the heterogeneous large-scale GDM with the individual concerns and satisfactions, IEEE Transactions on Fuzzy Systems. (in press, doi: 10.1109/TFUZZ.2017.2697403).

[68] Z. Zhang, C. Guo, L. Martínez, Managing multi-granular linguistic distribution assessments in large-scale multi-attribute group decision making, IEEE Transactions on Systems, Man, and Cybernetics: Systems 47 (2017) 3063 - 3076.

[69] S. Zadrozny, J. Kacprzyk, An internet-based group decision and consensus reaching support system, In: X. Yu, J. Kacprzyk (eds) Applied decision support with soft computing, Springer, Heidelberg (2003) 263-275.

[70] S. Zadrozny, An approach to the consensus reaching support in fuzzy environment. In: J. Kacprzyk, H. Nurmi, M. Fedrizzi (eds.) Consensus under fuzziness, Kluwer, Boston (1997) 83-109.

[71] J.J. Zhu, S.T. Zhang, Y. Chen, L.L. Zhang, A hierarchical clustering approach based on three-dimensional gray relational analysis for clustering a large group of decision makers with double information, Group Decision and Negotiation 25 (2016) 325-354. 